\title{
Vibration analysis of rotating rings with complex support stiffnesses
}

\author{
Fuchun Yang ${ }^{1, a}$, Yue Zhang ${ }^{1}$ and Hailong $\mathrm{Li}^{1}$ \\ ${ }^{1}$ School of Mechanical Engineering, Shandong University, Jinan 250061, China
}

\begin{abstract}
Vibration characteristics of rotating rings with complex support stiffnesses are studied. The complex stiffnesses of the rotating ring include discrete stiffnesses and partially distributed stiffnesses. The governing equations are established by Hamilton's principle. The governing equations are cast in matrix differential operators and discretized using Galerkin's method. The eigenvalue problem is dealt with state space matrix and the natural frequencies and vibration modes are obtained. The properties of natural frequencies and vibration modes of rotating rings are studied. The results illustrate that frequency separation and frequency veering happen with the increase of rotation speed. The vibration modes are not dominated by only one nodal diameter while dominated by several nodal diameters because the discrete and partially distributed stiffnesses disrupt the axisymmetry of rotating rings. The influences of several parameters to vibration properties of rotating rings are also investigated.
\end{abstract}

\section{Introduction}

Vibration properties of rotating rings are investigated by researchers for different structures, for instance, rotors, bearings, gears, etc. This work is motivated by star-type planetary gear sets, where rotating gears often have a thin rim [1], especially in aerospace applications. The rotating rings are supported by both discrete stiffnesses of mesh pairs and partially distributed stiffnesses of support bearings and these complex supports may cause quite different vibration properties compared to free rotating rings.

Vibrations of rotating rings have been investigated by many researchers. Bert and Chen [2] studied the in-plane and out-plane vibration of rotating rings on an elastic foundation. Free vibrations of rings on rigid radial supports were investigated by Rao and Sundararajan [3], where the ring was separated into several parts with supports located at the ends of each segment. Carrier [4] derived the governing equations for rotating rings in a rotating basis. Endo et al. [5] investigated different strain measures in the derivation of the equation of motion. Bickford and Reddy [6] included shear deformation and rotary inertia in their derivation. Reddy and Bickford [7] studied the vibration of rotating rings with equally spaced discrete stiffnesses. Huang and Soedel [8] derived a model using Lagrangian strain. They calculated natural frequencies for varying rotation speeds and find the dynamic response. Nackenhorst and Brinkmeier [9] used two observer systems, that is, Lagrangian and Eulerian systems, to derive rotating ring models. Wu and Parker [10] studied the vibration of rings on a general elastic foundation. There are fewer studies on the vibration of rotating rings with discrete supports. Canchi

\footnotetext{
a Corresponding author: yfc26@163.com
} 
and Parker [11] studied the parametric instability of rotating rings connected to moving discrete springs with time-varying stiffness. Cooley and Parker [12] studied the vibration of high-speed rotating rings coupled to space-fixed stiffnesses.

As mentioned above, the support mode of bearings has not excited the attention of researchers and there has been no study on the vibration of rotating rings with discrete stiffnesses and partially distributed stiffnesses. In this work, the work will mainly focus on vibration properties of rotating rings with complex support stiffnesses.

\section{Vibration model of rotating ring}

The vibration model of a rotating ring with complex support stiffnesses is shown in Figure1. The rotating speed is $\omega$. The ring-fixed basis is $\{\mathbf{i}, \mathbf{j}\}$ and the $\left\{\mathbf{e}_{1}, \mathbf{e}_{2}\right\}$ basis is a local basis on the middle surface of the ring, where $u(\theta, t)$ and $v(\theta, t)$ are radial and tangential deformations, respectively. $M$ is a material point on the middle surface and $\theta$ is the relative angle to the basis $\mathbf{i}$, where $0 \leq \theta \leq 2 \pi$. $R$ is the radii of the middle surface. The density of the ring is $\rho$, its elastic modulus is $E$, its section area is $A$. $k_{m 1}$ and $k_{m 2}$ are discrete stiffnesses in tangential direction. $k_{b}$ is the partially distributed stiffnesses between $\theta_{b 1}$ and $\theta_{b 2}$.

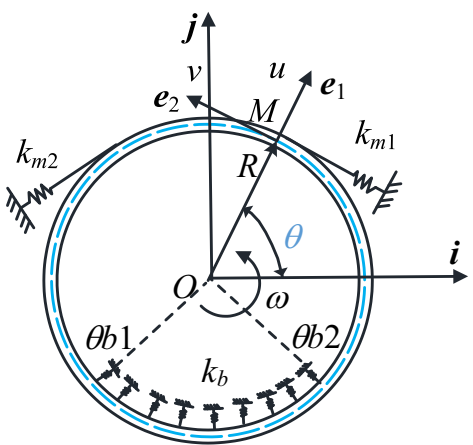

Figure 1. vibration model of a rotating ring with complex support stiffnesses.

By applying the kinetics principles, the kinetic energy of the ring is obtained as

$$
K_{E}=\frac{1}{2} \rho A R_{1} \int_{0}^{2 \pi}\left[(\dot{u}-\omega v)^{2}+(\dot{v}+\omega u+R \omega)\right] \mathrm{d} \theta
$$

The strain energy of the ring is derived using the engineering strain as

$$
S_{e}=\frac{1}{2} \iint_{A \theta} \sigma \varepsilon d A R_{1} \mathrm{~d} \theta=\frac{1}{2} \int_{0}^{2 \pi}\left[\frac{E A}{R_{1}}(u+\partial v / \partial \theta)^{2}+\frac{E I}{R_{1}^{3}}\left(\partial v / \partial \theta-\partial^{2} u / \partial \theta^{2}\right)^{2}\right] \mathrm{d} \theta
$$

The potential energy of discrete and partially distributed stiffnesses is

$$
S_{s}=\sum_{i=1}^{N b} \frac{1}{2} k_{b}\left[u \delta\left(\theta-\theta_{b i}\right)\right]^{2}+\sum_{i=1}^{N p} \frac{1}{2} k_{m i}\left[v \delta\left(\theta-\theta_{p i}\right)\right]^{2}
$$

Accordingly, the governing equations can be derived by Hamilton's principle as

$$
\left\{\begin{array}{l}
\rho A R \frac{\partial^{2} u}{\partial t^{2}}-2 \rho A R \omega \frac{\partial v}{\partial t}-\rho A R \omega^{2} u+\frac{E A}{R}\left(u+\frac{\partial v}{\partial \theta}\right)-\frac{E I}{R^{3}}\left(\frac{\partial^{3} v}{\partial \theta^{3}}-\frac{\partial^{4} u}{\partial \theta^{4}}\right)+\sum_{i=1}^{N b} k_{b} u \delta\left(\theta-\theta_{b i}\right)=0 \\
\rho A R \frac{\partial^{2} v}{\partial t^{2}}+2 \rho A R \omega \frac{\partial u}{\partial t}-\rho A R \omega^{2} v-\frac{E A}{R}\left(\frac{\partial u}{\partial \theta}+\frac{\partial^{2} v}{\partial \theta^{2}}\right)-\frac{E I}{R^{3}}\left(\frac{\partial^{2} v}{\partial \theta^{2}}-\frac{\partial^{3} u}{\partial \theta^{3}}\right)+\sum_{i=1}^{N p} k_{m i} v \delta\left(\theta-\theta_{p i}\right)=0
\end{array}\right.
$$

To investigate the general properties of rotating rings, Equation (1) is normalized by the following variables 


$$
\omega_{0}=\frac{1}{R_{1}^{2}} \sqrt{\frac{E I}{\rho A}}, \quad \bar{u}, \bar{v}=\frac{u, v}{R_{1}}, \bar{t}=\omega_{0} t, \bar{\omega}, \bar{\Omega}=\frac{\omega, \Omega}{\omega_{0}}, \bar{k}_{m p}, \bar{k}_{b}=\frac{k_{m p}, k_{b} R_{1}^{3}}{E I}, \gamma=\frac{E A R_{1}^{2}}{E I}
$$

The dimensionless equations are obtained by substitution of the above normalized variables to Equation (4) and (5), the equations are written as

$$
\left\{\begin{array}{l}
\frac{\partial^{2} u}{\partial t^{2}}-2 \omega \frac{\partial v}{\partial t}-\omega^{2} u+\gamma\left(u+\frac{\partial v}{\partial \theta}\right)-\left(\frac{\partial^{3} v}{\partial \theta^{3}}-\frac{\partial^{4} u}{\partial \theta^{4}}\right)+\sum_{i=1}^{N b} k_{b} u \delta\left(\theta-\theta_{b}\right)=0 \\
\frac{\partial^{2} v}{\partial t^{2}}+2 \omega \frac{\partial u}{\partial t}-\omega^{2} v-\gamma\left(\frac{\partial u}{\partial \theta}+\frac{\partial^{2} v}{\partial \theta^{2}}\right)-\left(\frac{\partial^{2} v}{\partial \theta^{2}}-\frac{\partial^{3} u}{\partial \theta^{3}}\right)+\sum_{i=1}^{N p} k_{m p} v \delta\left(\theta-\theta_{p}\right)=0
\end{array}\right.
$$

In Equation (7) and (8) the bar on variables are removed for convenience and Equation (7) and (8) are rewritten in matrix form by partial differential operators as

$$
\mathbf{M} \ddot{\mathbf{x}}+\omega \mathbf{G} \dot{\mathbf{x}}+\mathbf{K x}=\mathbf{0}
$$

where $\mathbf{x}=[\mathrm{u} \mathrm{v}]^{\mathrm{T}}$ and $\mathbf{M}, \mathbf{G}, \mathbf{K}$ are the corresponding matrix. In order to analyse the vibration of the rotating ring, Equation (5) is discretized using Galerkin's method by choosing basis functions,

$$
\mathbf{x}=\left[\sum_{n=-N}^{N} c_{n} \mathrm{e}^{j n \theta} \quad \sum_{m=-M}^{M} d_{m} \mathrm{e}^{j m \theta}\right]^{T} \mathrm{e}^{\lambda t}
$$

Then the eigenvalue equations about $c_{n}$ and $d_{m}$ are obtained and it is solved following the state space eigenvalue problem in reference [13].

\section{Results}

Since this work is motivated by planetary gear sets, the parameters of a gear ring in a planetary gear system are given in Table 1 .

Table 1. Parameters of a rotating ring.

\begin{tabular}{lc}
\hline Ring radius $R(\mathrm{~mm})$ & 80 \\
Cross section area $A\left(\mathrm{~mm}^{2}\right)$ & 250 \\
Area moment of inertia $I\left(\mathrm{~mm}^{4}\right)$ & 2083 \\
Elastic modulus $E(\mathrm{GPa})$ & 206 \\
Density $\rho\left(\mathrm{kg} / \mathrm{m}^{3}\right)$ & 7800 \\
Mean mesh stiffness $k_{m p}(\mathrm{~N} / \mathrm{m})$ & $5 \times 10^{8}$ \\
Support stiffness $k_{b}(\mathrm{~N} / \mathrm{m})$ & $1 \times 10^{8}$ \\
\hline
\end{tabular}

For each nodal diameter there are positive and negative natural frequencies and modes that correspond to forward and backward waves, respectively. In the following analysis only the positive frequency properties are illustrated since the positive and negative frequencies have the same magnitude but opposite sign.

Figure $2 \mathrm{a}$ shows the natural frequencies of a rotating ring with complex support stiffnesses calculated by the Galerkin method. As shown in Figure 2a, for the stationary system the eigenvalues have degeneracy two, which is typical in axisymmetric spinning systems, such as shafts, disk-spindle system. For non-zero rotation speeds, the eigenvalues separate, becoming district two frequencies which corresponding to forward and backward waves, respectively. The natural frequencies increase with the rotating speed. And in high frequency areas, frequency veering occurs drastically which means the vibration mode exchange at these areas drastically, but for the lower order frequencies there is no frequency veering and the rotation speeds affect the frequencies slightly. In addition, there are no 
critical speeds and instability for the system since the frequencies are purely imaginary and never reach to zero in the analysis speed range.

It can be seen from Figure $2 \mathrm{~b}$ that $c_{n}$ and $d_{m}$ are comprised of several components of lower orders and there is no district dominated component. This means that since the complex support stiffnesses with discrete and partially distributed stiffnesses disrupt the axisymmetry of the rotating ring, the vibration modes are not dominated by only one nodal diameter anymore as free rings does, but dominated by several nodal diameters. And in the lower order modes, the partially distributed stiffnesses affect the vibration mode heavily.

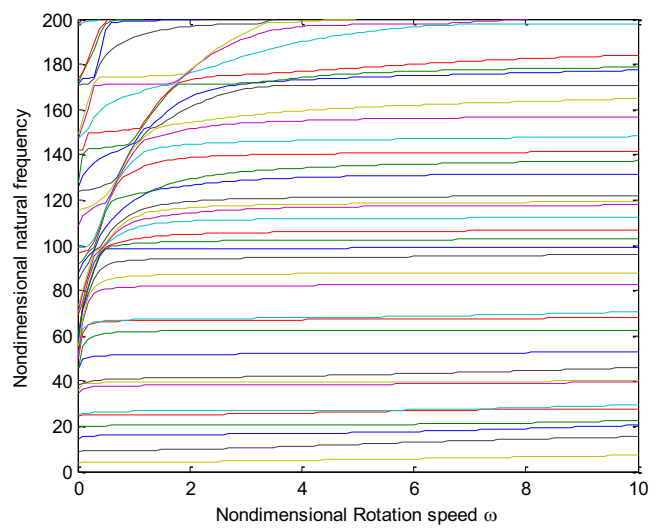

(a)

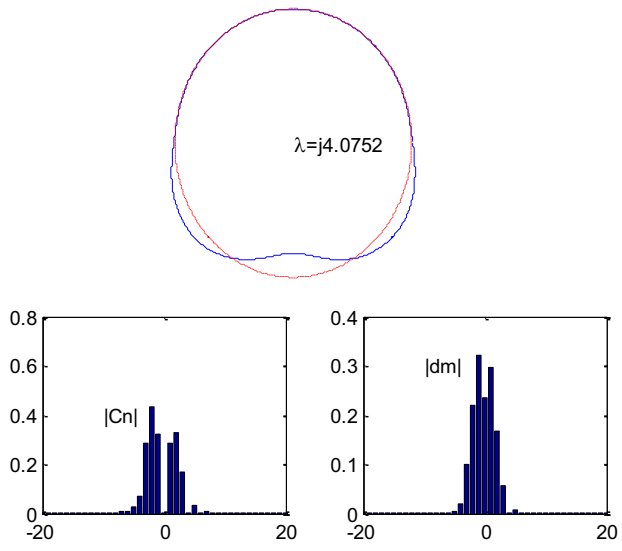

(b)

Figure 2. (a) Natural frequencies vs. rotating speed; (b) representative vibration mode and its components at speed $\omega=2$ (dash line-original position, solid line-vibration mode).

Figure 3a illustrates the influence of partially distributed stiffnesses to the natural frequencies. It can be seen that almost all the frequencies are affected by the partial distributed stiffnesses, but the intermediate or higher frequencies are affected heavily while the lower frequencies are affected slightly. And in the analysis range of support stiffness in Figure 3a, frequency veering also happens with the increase of the stiffnesses.

Figure $3 \mathrm{~b}$ shows the influence of discrete stiffnesses to the natural frequencies. As seen in Figure $3 \mathrm{~b}$ a majority of frequencies are affected slightly by the discrete stiffness and only the intermediate frequencies and partial high frequencies are affected heavily. And frequency veering also exists in certain areas with the increase of discrete stiffnesses.

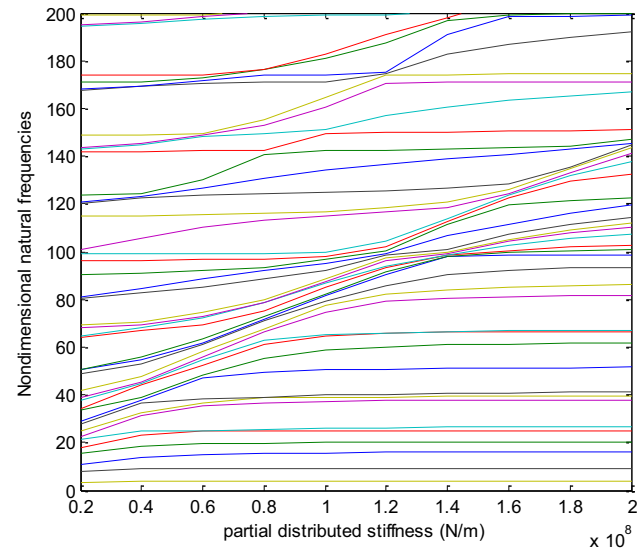

(a)

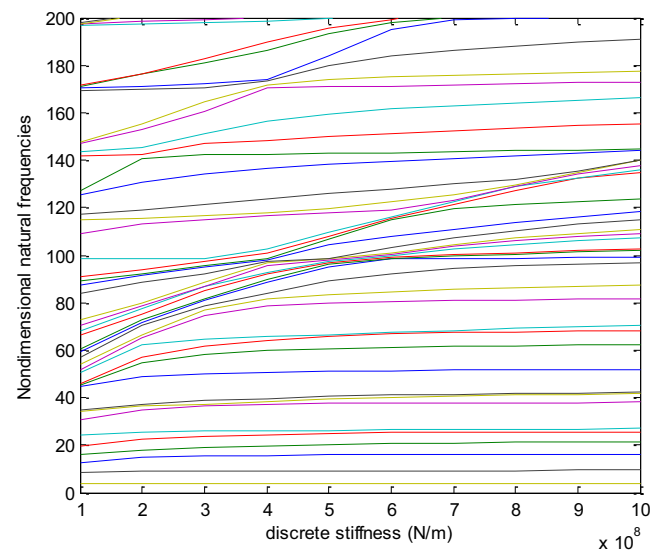

(b)

Figure 3. Influence of stiffnesses to natural frequencies (a) partial distributed stiffness and (b) discrete stiffness to natural frequencies at $\omega=2$. 


\section{Conclusions}

This paper established the governing equation of a rotating ring with complex support stiffnesses by Hamilton's principle. The governing equation is treated in matrix form and the eigenvalue problem is solved using Galerkin's method. The complex support stiffnesses change the vibration properties compared to free rotating rings. The phenomenon of frequency veering occurs with the increase of rotation speed. The influences of partially distributed and discrete stiffnesses to natural frequencies and naturel mode were also investigated. The results show that frequency separation and frequency veering happen with the increase of rotation speed. The vibration modes are not dominated by only one nodal diameter while dominated by several nodal diameters because the discrete and partially distributed stiffnesses disrupt the axisymmetry of rotating rings. The changes of the discrete or partially distributed stiffnesses mainly affect the intermediate frequencies and with the increase to stiffnesses the frequency veering also happens.

\section{Acknowledgements}

This work was supported by the National Natural Science Foundation of China (Grant numbers 51775309) and Young Scholars Program of Shandong University.

\section{References}

1. A. Kapelevich, Y. Shekhtman. Intl. Conf. on Gear, Munich, Germany, (2017)

2. C. Bert, L. Chen. J. Sound Vib., 122 (1988)

3. S. Rao, V. Sundararajan. J. Appl. Mech., 36 (1969)

4. G. Carrier. Q. Appl. Math., 3 (1945)

5. M. Endo, K. Hatamura, M. Sakata, O. Taniguchi. J. Sound Vib., 92 (1984)

6. W. Bickford, E. Reddy. J. Sound Vib., 101 (1985)

7. E. Reddy, W. Bickford. J. Sound Vib., 103 (1985)

8. E. Huang, W. Soedel. J. Sound Vib., 115 (1987)

9. U. Nackenhorst, M. Brinkmeier. Arch. Appl. Mech., 78 (2008)

10. X. Wu, R. Parker. J. Sound Vib., 295 (2006)

11. S. Canchi, R. Parker. J. Vib. Acous., 128 (2006)

12. C. Cooley, R. Parker. J. Sound Vib., 333 (2014)

13. L. Meirovitch. AIAA Journal, 12 (1974). 